\title{
On Multiple Convolutions and Time Scales
}

\author{
Hassan Eltayeb, ${ }^{1}$ Adem Kılıçman, ${ }^{2}$ and Brian Fisher ${ }^{3}$ \\ ${ }^{1}$ Mathematics Department, College of Science, King Saud University, P.O. Box 2455, Riyadh 11451, Saudi Arabia \\ ${ }^{2}$ Department of Mathematics and Institute for Mathematical Research, Universiti Putra Malaysia (UPM), \\ 43400 Serdang, Selangor, Malaysia \\ ${ }^{3}$ Department of Mathematics, University of Leicester, Leicester LE1 7RH, UK
}

Correspondence should be addressed to Adem Kılıçman; akilic@upm.edu.my

Received 23 September 2013; Accepted 7 November 2013

Academic Editor: Suares Clovis Oukouomi Noutchie

Copyright (C) 2013 Hassan Eltayeb et al. This is an open access article distributed under the Creative Commons Attribution License, which permits unrestricted use, distribution, and reproduction in any medium, provided the original work is properly cited.

The properties of the multiple Laplace transform and convolutions on a time scale are studied. Further, some related results are also obtained by utilizing the double Laplace transform. We also provide an example in order to illustrate the main result.

\section{Introduction}

A time scale $T$ is a nonempty closed subset of the real numbers $\mathbb{R}$ and it has the topology that it inherits from the real numbers with the standard topology. It is also well known that if the time scale is the set of real numbers, then the dynamic equation is a differential equation, whilst if the time scale is the set of integers, then the dynamic equation is reduced to a difference equation. Thus integral transform of differential and integral calculus on time scales allows us to develop a theory of differential equations. For the single Laplace transform on time scales, see [1-3]. Similarly, in [4-8], the authors defined the time scale to be any nonempty closed subset of the real numbers and provided the motivation and formulation of delta derivatives on a time scale as well as the properties of delta derivatives and integrals. In [2], Bohner and Peterson defined the Laplace transform of a time scale for function of single variable as follows.

Definition 1. For $f: \top \rightarrow \mathbb{C}$, the time scale or generalized Laplace transform of $f$, denoted by $L[f(t)]$ or $F(z)$, is given by

$$
L[f(t)](z)=\int_{0}^{\infty} e_{\ominus z}^{\sigma}(t, 0) f(t) \Delta t, \quad \text { for } z \in D\{f\},
$$

where $D\{f\}$ consists of all $z \in \mathbb{C}$ for which the improper integral exists and $1+\mu_{1} z \neq 0$ for all $t \in \mathrm{T}$.
Definition 2. The function $f: T \rightarrow \mathbb{R}$ is said to be of exponential type I if there exist constants $M, c>0$ such that $|f(t)| \leq M e^{c t}$. Furthermore, $f$ is said to be of exponential type II if there exist constants $M, c>0$ such that $|f(t)| \leq$ $M e_{c}(t, 0)$.

The following two theorems were proved in [3].

Theorem 3. The integral $\int_{0}^{\infty} e_{\ominus z}^{\sigma}(t, 0) f(t) \Delta t$ converges absolutely for $z \in D$ if $f(t)$ is of exponential type II with exponential constant c. For more details, see [3].

Theorem 4. Let $\alpha \in \mathbb{R}$ be regressive. Then

(I)

$$
L\left\{e_{\alpha}(t, 0)\right\}(z)=\frac{1}{z-\alpha},
$$

provided that $\lim _{t \rightarrow \infty} e_{\alpha \ominus z}(t, 0)=0$,

(II)

$$
L\left\{\cos _{\alpha}(t, 0)\right\}(z)=\frac{z}{z^{2}+\alpha^{2}},
$$

provided that $\lim _{t \rightarrow \infty} e_{i \alpha \ominus z}(t, 0)=\lim _{t \rightarrow \infty} e_{-i \alpha \ominus z}(t, 0)=0$, (III)

$$
L\left\{\sin _{\alpha}(t, 0)\right\}(z)=\frac{\alpha}{z^{2}+\alpha^{2}},
$$

provided that $\lim _{t \rightarrow \infty} e_{i \alpha \ominus z}(t, 0)=\lim _{t \rightarrow \infty} e_{-i \alpha \ominus z}(t, 0)=0$. 
The following theorem was also studied by Bohner and Peterson in [2].

Theorem 5. If $f: T \rightarrow \mathbb{R}$ is such that $f^{\Delta}$ is regulated, then

$$
L\left[f^{\Delta}\right](z)=z L[f(t)](z)-f(0),
$$

for all $z \in D\{f\}$ such that $\lim _{t \rightarrow \infty}\left\{e_{\ominus z}(t) f(t)\right\}=0$.

Throughout the study, the following notation will be useful, where $T_{1}, T_{2}$ are time scales and

$$
\begin{gathered}
e_{a \oplus b}\left(t_{1}, t_{2}\right)=e_{b}\left(t_{1}\right) e_{b}\left(t_{2}\right), \\
e_{\ominus z_{1} \ominus z_{2}}\left(t_{1}, t_{2}\right)=e_{\ominus z_{1}}\left(t_{1}\right) e_{\ominus z_{2}}\left(t_{2}\right) .
\end{gathered}
$$

In [2], further it was proved that

$$
e_{\ominus z}^{\sigma}=\frac{e_{\ominus z_{1}}}{(1+\mu z)}
$$

was satisfied, where $1+\mu_{1} z \neq 0$, by using the property

$$
e_{z}^{\sigma}=(1+\mu z) e_{z}, \quad \ominus z=-\frac{z}{1+z}, \quad p \ominus q=\frac{p-q}{(1+\mu q)} \text {. }
$$

Later in [9], Bohner and Guseinov defined the single convolution operation on time scale of two functions $f, g$ : $\top \rightarrow \mathbb{R}$ by the following formula:

$$
(f * g)(t)=\int_{t_{0}}^{t} \hat{f}(t, \sigma(s)) g(s) \Delta s .
$$

In this study, we introduce double Laplace transform on time scales and study some of the properties in solving partial differential equations. Further, we also extend the $\Delta$-Laplace transform, which is given by Jackson in [10], to the multiple $\Delta$-Laplace transform as follows.

Definition 6. Let $0 \in \mathrm{T}_{1}, \mathrm{~T}_{2}$, $\sup \left\{\mathrm{T}_{1}, \mathrm{~T}_{2}\right\}=\infty$, and $t_{1} \in$ $\mathrm{T}_{1}, t_{2} \in \mathrm{T}_{2}$. The $\Delta$-double Laplace transform of the function $f\left(t_{1}, t_{2}\right)$ (for $f \in C\left(\mathrm{~T}_{1} \times \mathrm{T}_{2}\right)$ ) with respect to $t_{1}, t_{2}$ is given by

$$
\begin{aligned}
& L_{t_{1}} L_{t_{2}}\left[f\left(t_{1}, t_{2}\right)\right]\left(z_{1}, z_{2}\right) \\
& \quad=\iint_{0}^{\infty} e_{\ominus z_{1} \ominus z_{2}}^{\sigma_{1} \sigma_{2}}\left(t_{1}, t_{2}\right) f\left(t_{1}, t_{2}\right) \Delta_{1} t_{1} \Delta_{2} t_{2}
\end{aligned}
$$

provided that the integral exists, further $\left(1+\mu_{1} z_{1}\right) \neq 0$, and $\left(1+\mu_{2} z_{2}\right) \neq 0$, for all $\left(t_{1}, t_{2}\right) \in\left(T_{1} \times T_{2}\right)_{+}$.

Lemma 7. If $z_{1}, z_{2} \in \mathbb{C}$ are regressive, then

$$
e_{\ominus z_{1} \ominus z_{2}}^{\sigma_{1} \sigma_{2}}=\frac{e_{\ominus z_{1} \ominus z_{2}}}{\left(1+\mu_{1} z_{1}\right)\left(1+\mu_{2} z_{2}\right)} .
$$

Proof. By using the above analysis, we have

$$
\begin{aligned}
e_{\ominus z_{1} \ominus z_{2}}^{\sigma_{1} \sigma_{2}} & =\left(\left(1+\mu_{1}\left(\ominus z_{1}\right)\right)\right) e_{\ominus z_{1}}\left(1+\mu_{2}\left(\ominus z_{2}\right)\right) e_{\ominus z_{2}} \\
& =\left[1-\mu_{1} \frac{z_{1}}{1+\mu_{1} z_{1}}\right]\left[1-\mu_{2} \frac{z_{2}}{1+\mu_{2} z_{2}}\right] e_{\ominus z_{1} e_{\ominus z_{2}}} \\
& =\frac{e_{\ominus z_{1} \ominus z_{2}}}{\left(1+\mu_{1} z_{1}\right)\left(1+\mu_{2} z_{2}\right)} .
\end{aligned}
$$

As an example, we can easily compute $L_{t_{2}} L_{t_{1}}\left[e_{a \oplus b}\left(t_{1}\right.\right.$, $\left.\left.t_{2}, 0,0\right)\right]\left(z_{1}, z_{2}\right)$, where $a, b$ are constants such that $\lim _{t_{1} \rightarrow \infty} e_{a \oplus b \ominus z_{1} \ominus z_{2}}\left(t_{1}, t_{2}, 0,0\right)=0$.

We have

$$
\begin{aligned}
& L_{t_{2}} L_{t_{1}}\left[e_{a \oplus b}\left(t_{1}, t_{2}, 0,0\right)\right]\left(z_{1}, z_{2}\right) \\
& =\iint_{0}^{\infty} e_{a \oplus b}\left(t_{1}, t_{2}, 0,0\right) e_{\ominus z_{1} \ominus z_{2}}^{\sigma_{1} \sigma_{2}}\left(t_{1}, t_{2}\right) \Delta_{1} t_{1} \Delta_{2} t_{2} \\
& =\iint_{0}^{\infty}\left(1+\mu_{\sigma_{1}}\left(t_{1}\right) \ominus z_{1}\left(t_{1}\right)\right)\left(1+\mu_{\sigma_{2}}\left(t_{2}\right) \ominus z_{2}\left(t_{2}\right)\right) e_{a \oplus b} \\
& \times\left(t_{1}, t_{2}, 0,0\right) e_{\ominus z_{1} \ominus z_{2}}\left(t_{1}, t_{2}\right) \Delta_{1} t_{1} \Delta_{2} t_{2} \\
& =\int_{0}^{\infty}\left(1-\frac{\mu_{\sigma_{1}}\left(t_{1}\right) z_{1}}{1+\mu_{\sigma_{1}} \ominus z_{1}}\right) \\
& \times\left[\int_{0}^{\infty}\left(1-\frac{\mu_{\sigma_{1}}\left(t_{2}\right) z_{2}}{1+\mu_{\sigma_{2}} \ominus z_{2}}\right) e_{b \ominus z_{2}}\left(t_{1}, t_{2}, 0,0\right) \Delta_{2} t_{2}\right] e_{a \ominus z_{1}} \\
& \times\left(t_{1}, t_{2}\right) \Delta_{1} t_{1} \\
& =\int_{0}^{\infty}\left(\frac{1}{1+\mu_{\sigma_{1}} \ominus z_{1}}\right) \\
& \times\left[\int_{0}^{\infty}\left(\frac{1}{1+\mu_{\sigma_{2}} \ominus z_{2}}\right) e_{b \ominus z_{2}}\left(t_{1}, t_{2}, 0,0\right) \Delta_{2} t_{2}\right] \\
& \times e_{a \ominus z_{1}}\left(t_{1}, t_{2}, 0,0\right) \Delta_{1} t_{1} \\
& =\frac{1}{a-z_{1}} \int_{0}^{\infty}\left[\frac{1}{b-z_{2}} \int_{0}^{\infty} b \ominus z_{2} e_{b \ominus z_{2}}\left(t_{1}, t_{2}, 0,0\right) \Delta_{2} t_{2}\right] \\
& \times a \ominus z_{1} e_{a \ominus z_{1}}\left(t_{1}, t_{2}, 0,0\right) \Delta_{1} t_{1} \\
& =\frac{1}{a-z_{1}} \int_{0}^{\infty}\left[\frac{1}{b-z_{2}} \int_{0}^{\infty}\left(e_{b \ominus z_{2}}\left(t_{1}, t_{2}, 0,0\right)\right)_{\Delta_{2} t_{2}} \Delta_{2} t_{2}\right] \\
& \times\left(e_{a \ominus z_{1}}\left(t_{1}, t_{2}, 0,0\right)\right)_{\Delta_{1} t_{1}} \Delta_{1} t_{1} \\
& =\frac{1}{\left(z_{1}-a\right)\left(z_{2}-b\right)} .
\end{aligned}
$$

For $t_{1} \in \mathrm{T}_{1}$ and $t_{2} \in \mathrm{T}_{2}$ with $t_{1} \geq \alpha$ and $t_{2} \geq \beta$, we can easily see that

$$
\begin{aligned}
& \cosh _{(\alpha \oplus \beta)}\left(t_{1}, t_{2}\right)=\frac{e_{\left(\alpha t_{1} \oplus \beta t_{2}\right)}+e_{-\left(\alpha t_{1} \oplus \beta t_{2}\right)}}{2}, \\
& \sinh _{(\alpha \oplus \beta)}\left(t_{1}, t_{2}\right)=\frac{e_{\left(\alpha t_{1} \oplus \beta t_{2}\right)}-e_{-\left(\alpha t_{1} \oplus \beta t_{2}\right)}}{2}, \\
& \cos _{(\alpha \oplus \beta)}\left(t_{1}, t_{2}\right)=\frac{e_{i\left(\alpha t_{1} \oplus \beta t_{2}\right)}+e_{-i\left(\alpha t_{1} \oplus \beta t_{2}\right)}}{2}, \\
& \sin _{(\alpha \oplus \beta)}\left(t_{1}, t_{2}\right)=\frac{e_{i\left(\alpha t_{1} \oplus \beta t_{2}\right)}-e_{-i\left(\alpha t_{1} \oplus \beta t_{2}\right)}}{2 i},
\end{aligned}
$$


and then the double Laplace transforms are given by

$$
\begin{aligned}
L_{t_{2}} & L_{t_{1}}\left[\cosh _{(\alpha \oplus \beta)}\left(t_{1}, t_{2}\right)\right] \\
& =\frac{1 /\left(\left(z_{1}-\alpha\right)\left(z_{2}-\beta\right)\right)+1 /\left(\left(z_{1}+\alpha\right)\left(z_{2}+\beta\right)\right)}{2} \\
& =\frac{z_{1} z_{2}+\alpha \beta}{\left(z_{2}^{2}-\beta^{2}\right)\left(z_{1}^{2}-\alpha^{2}\right)}, \\
L_{t_{2}} & L_{t_{1}}\left[\sinh _{(\alpha \oplus \beta)}\left(t_{1}, t_{2}\right)\right] \\
& =\frac{1 /\left(\left(z_{1}-\alpha\right)\left(z_{2}-\beta\right)\right)-1 /\left(\left(z_{1}+\alpha\right)\left(z_{2}+\beta\right)\right)}{2} \\
& =\frac{\beta z_{1}+\alpha z_{2}}{\left(z_{2}^{2}-\beta^{2}\right)\left(z_{1}^{2}-\alpha^{2}\right)} .
\end{aligned}
$$

Similarly, we have

$$
\begin{aligned}
& L_{t_{2}} L_{t_{1}}\left[\cos _{(\alpha \oplus \beta)}\left(t_{1}, t_{2}\right)\right]=\frac{z_{1} z_{2}-\alpha \beta}{\left(z_{2}^{2}+\beta^{2}\right)\left(z_{1}^{2}+\alpha^{2}\right)}, \\
& L_{t_{2}} L_{t_{1}}\left[\sin _{(\alpha \oplus \beta)}\left(t_{1}, t_{2}\right)\right]=\frac{z_{1} z_{2}+\alpha \beta}{\left(z_{2}^{2}+\beta^{2}\right)\left(z_{1}^{2}+\alpha^{2}\right)} .
\end{aligned}
$$

Proposition 8. Suppose $T_{1}, T_{2}$ are two time scales such that $\mu_{1}<N_{1}$ and $\mu_{1}<N_{2}$ for some $N_{1}, N_{2} \in \mathbb{R}$ and all $\left(t_{1}, t_{2}\right) \in$ $\mathrm{T}_{1} \times \mathrm{T}_{2}$. Let $f:\left(\mathrm{T}_{1} \times \mathrm{T}_{2}\right)_{+} \rightarrow \mathbb{R}$ and

$$
\lim _{\substack{t_{1} \rightarrow \infty \\ t_{2} \rightarrow \infty}}\left\{e_{\ominus z_{1} \ominus z_{2}}\left(t_{1}, t_{2}\right) f\left(\sigma\left(t_{1}\right), \sigma\left(t_{2}\right), 0,0\right)\right\}=0 .
$$

Then

$$
\lim _{\substack{t_{1} \rightarrow \infty \\ t_{2} \rightarrow \infty}}\left\{e_{\ominus z_{1} \ominus z_{2}}\left(t_{1}, t_{2}\right) f\left(t_{1}, t_{2}, 0,0\right)\right\}=0 .
$$

Proof. Since

$$
\lim _{\substack{t_{1} \rightarrow \infty \\ t_{2} \rightarrow \infty}}\left\{e_{\ominus z_{1} \ominus z_{2}}\left(t_{1}, t_{2}\right) f\left(\sigma\left(t_{1}\right), \sigma\left(t_{2}\right), 0,0\right)\right\}=0
$$

and on using the following relation:

$$
\begin{aligned}
& e_{\ominus p_{1} \ominus p_{2}}\left(\sigma\left(t_{1}\right), \sigma\left(t_{2}\right), 0,0\right) \\
& =\frac{1}{\left(1+\mu_{1} p_{1}\right)\left(1+\mu_{2} p_{2}\right)} e_{\ominus p_{1} \ominus p_{2}}\left(t_{1}, t_{2}, 0,0\right) \\
& =\frac{\ominus p_{1} \ominus p_{2}}{p_{1} p_{2}} e_{\ominus p_{1} \ominus p_{2}}\left(t_{1}, t_{2}, 0,0\right),
\end{aligned}
$$

we have

$$
\begin{gathered}
\lim _{\substack{t_{1} \rightarrow \infty \\
t_{2} \rightarrow \infty}}\left\{\frac{\ominus z_{1}\left(t_{1}\right) \ominus z_{2}\left(t_{2}\right)}{z_{1} z_{2}} e_{\ominus z_{1} \ominus z_{2}}\left(t_{1}, t_{2}\right) f\left(t_{1}, t_{2}, 0,0\right)\right\}=0, \\
\lim _{\substack{t_{1} \rightarrow \infty \\
t_{2} \rightarrow \infty}}\left\{\frac{\ominus z_{1}\left(t_{1}\right) \ominus z_{2}\left(t_{2}\right)}{z_{1} z_{2}\left(1+\mu_{1} z_{1}\right)\left(1+\mu_{2} z_{2}\right)} e_{\ominus z_{1} \ominus z_{2}}\right. \\
\left.\times\left(t_{1}, t_{2}\right) f\left(t_{1}, t_{2}, 0,0\right)\right\}=0, \\
\lim _{\substack{t_{1} \rightarrow \infty \\
t_{2} \rightarrow \infty}}\left\{\frac{1}{\left(1+\mu_{1} z_{1}\right)\left(1+\mu_{2} z_{2}\right)} e_{\ominus z_{1} \ominus z_{2}}\right. \\
\left.\times\left(t_{1}, t_{2}\right) f\left(t_{1}, t_{2}, 0,0\right)\right\}=0 .
\end{gathered}
$$

Since $\mu_{1}\left(t_{2}\right)<N_{1}$ and $\mu_{1}\left(t_{2}\right)<N_{2}$, we get

$$
\begin{aligned}
& \lim _{\substack{t_{1} \rightarrow \infty \\
t_{2} \rightarrow \infty}}\left|\left\{\frac{e_{\ominus z_{1} \ominus z_{2}}\left(t_{1}, t_{2}\right) f\left(t_{1}, t_{2}, 0,0\right)}{\left(1+\mu_{1} z_{1}\right)\left(1+\mu_{2} z_{2}\right)}\right\}\right| \\
& >\left|\frac{\lim _{t_{1} \rightarrow \infty}\left\{e_{\ominus z_{1} \ominus z_{2}}\left(t_{1}, t_{2}\right) f\left(t_{1}, t_{2}, 0,0\right)\right\}}{\left(1+N_{1} z_{1}\right)\left(1+N_{2} z_{2}\right)}\right|>0 .
\end{aligned}
$$

Thus we obtain that

$$
\begin{aligned}
& \frac{1}{\left(1+N_{1} z_{1}\right)\left(1+N_{2} z_{2}\right)} \\
& \quad \times \lim _{\substack{t_{1} \rightarrow \infty \\
t_{2} \rightarrow \infty}}\left\{e_{\ominus z_{1} \ominus z_{2}}\left(t_{1}, t_{2}\right) f\left(t_{1}, t_{2}, 0,0\right)\right\}=0
\end{aligned}
$$

and it follows that

$$
\lim _{\substack{t_{1} \rightarrow \infty \\ t_{2} \rightarrow \infty}}\left\{e_{\ominus z_{1} \ominus z_{2}}\left(t_{1}, t_{2}\right) f\left(t_{1}, t_{2}, 0,0\right)\right\}=0 .
$$

Theorem 9. Let $f:\left(T_{1} \times T_{2}\right)_{+} \rightarrow \mathbb{R}$ be regulated and let

$$
F\left(t_{1}, t_{2}\right)=\iint_{0}^{t_{1}} f(\zeta, \eta) \Delta \zeta \Delta \eta
$$

$$
\text { for }\left(t_{1}, t_{2}\right) \in\left(\mathrm{T}_{1} \times \mathrm{T}_{2}\right)_{+} \text {. Then }
$$

$$
\begin{aligned}
L_{t_{1}} L_{t_{2}}\left[f\left(t_{1}, t_{2}\right)\right]\left(z_{1}, z_{2}\right) & \\
= & \frac{1}{z_{1} z_{2}} L_{t_{1}} L_{t_{2}}\left[f\left(t_{1}, t_{2}\right)\right]\left(z_{1}, z_{2}\right) \\
& -\lim _{\substack{t_{1} \rightarrow \infty \\
t_{2} \rightarrow \infty}} e_{\ominus z_{1} \ominus z_{2}}\left(t_{1}, t_{2}\right) f\left(t_{1}, t_{2}, 0,0\right),
\end{aligned}
$$

for $z_{1}, z_{2} \neq 0$. 
Proof. By using the definition of the double Laplace transform on a time scale, we have

$$
\begin{aligned}
& L_{t_{1}} L_{t_{2}}\left[f\left(t_{1}, t_{2}\right)\right]\left(z_{1}, z_{2}\right) \\
& \quad=\iint_{0}^{\infty} e_{\ominus z_{1} \ominus z_{2}}^{\sigma_{1} \sigma_{2}}\left(t_{1}, t_{2}\right) F\left(t_{1}, t_{2}, 0,0\right) \Delta_{1} t_{1} \Delta_{2} t_{2},
\end{aligned}
$$

and on using (21), we have

$$
\begin{aligned}
& L_{t_{1}} L_{t_{2}}\left[f\left(t_{1}, t_{2}\right)\right]\left(z_{1}, z_{2}\right) \\
& =\frac{1}{z_{1} z_{2}} \iint_{0}^{\infty} e_{\ominus z_{1} \ominus z_{2}}\left(t_{1}, t_{2}\right)\left(\ominus z_{1} \ominus z_{2}\right) \\
& \quad \times F\left(t_{1}, t_{2}, 0,0\right) \Delta_{1} t_{1} \Delta_{2} t_{2} .
\end{aligned}
$$

Now, applying integration by parts, and on using the fact that $F(0,0)=0$ together with the fundamental theorem of calculus, we obtain that

$$
\begin{aligned}
L_{t_{1}} L_{t_{2}} & {\left[f\left(t_{1}, t_{2}\right)\right]\left(z_{1}, z_{2}\right) } \\
= & \frac{1}{z_{1} z_{2}} L_{t_{1}} L_{t_{2}}\left[f\left(t_{1}, t_{2}\right)\right]\left(z_{1}, z_{2}\right) \\
& \quad-\lim _{\substack{t_{1} \rightarrow \infty \\
t_{2} \rightarrow \infty}} e_{\ominus z_{1} \ominus z_{2}}\left(t_{1}, t_{2}\right) f\left(t_{1}, t_{2}, 0,0\right) .
\end{aligned}
$$

Example 10. Let $a \in \mathrm{T}_{1}, b \in \mathrm{T}_{2}, a, b>0$, and $H_{a, b}\left(t_{1}, t_{2}\right)=0$ for $t_{1} \in \mathrm{T}_{1}$ and $t_{2} \in \mathrm{T}_{2}, t_{1}<a$, and $t_{2}<b$ whilst $H_{a, b}\left(t_{1}, t_{2}\right)=1$ for $t_{1} \in \mathrm{T}_{1}, t_{2} \in \mathrm{T}_{2}$ and $t_{1} \geq a, t_{2} \geq b$, where $H_{a, b}\left(t_{1}, t_{2}\right)=H_{a}\left(t_{1}\right) \otimes H_{b}\left(t_{2}\right)$ and the symbol $\otimes$ denotes the tensor product. Then we have

$$
\begin{aligned}
L_{t_{1}} & L_{t_{2}}\left[H_{a, b}\left(t_{1}, t_{2}\right)\right]\left(z_{1}, z_{2}\right) \\
& =\iint_{0}^{\infty} e_{\ominus z_{1} \ominus z_{2}}^{\sigma_{1} \sigma_{2}}\left(t_{1}, t_{2}\right) H_{a, b}\left(t_{1}, t_{2}\right) \Delta_{1} t_{1} \Delta_{2} t_{2} \\
& =\frac{e_{\ominus z_{1}}(a) e_{\ominus z_{2}}(b)}{z_{1} z_{2}} .
\end{aligned}
$$

Now assume that $f\left(t_{1}, t_{2}\right): \top_{1} \times \top_{2} \rightarrow \mathbb{C}$ such that $f^{\Delta_{1}}$ is continuous. Then the $\Delta$-double Laplace transform

$$
L_{t_{1}} L_{t_{2}}\left[f^{\Delta_{1}}\left(t_{1}, t_{2}\right)\right]\left(z_{1}, z_{2}\right)=z_{1} \stackrel{\bar{F}}{F}\left(z_{1}, z_{2}\right)-\bar{F}\left(0, z_{2}\right)
$$

holds for those regressive $z_{1}, z_{2} \in \mathbb{C}$ with respect to $t_{1}$ and $t_{2}$ which further satisfies

$$
\lim _{\substack{t_{1} \rightarrow \infty \\ t_{2} \rightarrow \infty}}\left\{e_{\ominus z_{1} \ominus z_{2}}\left(t_{1}, t_{2}\right) f\left(t_{1}, t_{2}\right)\right\}=0 .
$$

We next extend the result that was proved by Davis et al. in [3].

Proposition 11. Let $(a, b) \in\left(\top_{1} \times \top_{2}\right)_{+}, a, b>0$. Assume that $f\left(t_{1}, t_{2}\right)$ is one of the following functions:

$$
\begin{gathered}
e_{a \oplus b}\left(t_{1}, t_{2}, 0,0\right), \quad \sinh _{(\alpha \oplus \beta)}\left(t_{1}+t_{2}\right), \\
\cosh _{(\alpha \oplus \beta)}\left(t_{1}+t_{2}\right), \quad \cos _{(\alpha \oplus \beta)}\left(t_{1}+t_{2}\right), \\
\text { or } \sin _{(\alpha \oplus \beta)}\left(t_{1}+t_{2}\right) .
\end{gathered}
$$

If $z_{1}, z_{2}, \alpha$, and $\beta$ are regressive and satisfy

$$
\begin{aligned}
& \lim _{\substack{t_{1} \rightarrow \infty \\
t_{2} \rightarrow \infty}}\left\{e_{\alpha \ominus z_{1} \beta \ominus z_{2}}\left(t_{1}, t_{2}\right) f\left(t_{1}, t_{2}, a, b\right)\right\} \\
& =\lim _{\substack{t_{1} \rightarrow \infty \\
t_{2} \rightarrow \infty}}\left\{e_{i \alpha \ominus z_{1} i \beta \ominus z_{2}}\left(t_{1}, t_{2}\right) f\left(t_{1}, t_{2}, a, b\right)\right\} \\
& =\lim _{\substack{t_{1} \rightarrow \infty \\
t_{2} \rightarrow \infty}}\left\{e_{-i \alpha \ominus z_{1}-i \beta \ominus z_{2}}\left(t_{1}, t_{2}\right) f\left(t_{1}, t_{2}, a, b\right)\right\},
\end{aligned}
$$

then

$$
\begin{aligned}
L_{t_{1}} & L_{t_{2}}\left[H_{a, b}\left(t_{1}, t_{2}\right) f\left(t_{1}, t_{2}, a, b\right)\right]\left(z_{1}, z_{2}\right) \\
& =e_{\ominus z_{1} \ominus z_{2}}(a, b, 0,0) L_{t_{1}} L_{t_{2}}\left[f\left(t_{1}, t_{2}, 0,0\right)\right] .
\end{aligned}
$$

Proof. Let us study the case $f\left(t_{1}, t_{2}, a, b\right)=e_{\alpha \oplus \beta}\left(t_{1}, t_{2}, a, b\right)$. By using (21), we get

$$
\begin{gathered}
e_{\alpha \oplus \beta}\left(t_{1}, t_{2}, a, b\right) e_{\ominus z_{1} \ominus z_{2}}\left(\sigma\left(t_{1}\right), \sigma\left(t_{2}\right), 0,0\right) \\
=\frac{e_{\alpha \oplus \beta}\left(t_{1}, t_{2}, a, b\right) e_{\ominus z_{1} \ominus z_{2}}\left(t_{1}, t_{2}, 0,0\right)}{\left(1+\mu_{1} z_{1}\right)\left(1+\mu_{2} z_{2}\right)}
\end{gathered}
$$

and by using Theorem 4 and (21) the right hand side of equation (37) is

$$
\begin{aligned}
= & \frac{e_{\ominus z_{1} \ominus z_{2}}(a, b, 0,0)}{\left(\alpha-z_{1}\right)\left(\beta-z_{2}\right)}\left(\frac{\left(\alpha-z_{1}\right)\left(\beta-z_{2}\right)}{\left(1+\mu_{1} z_{1}\right)\left(1+\mu_{2} z_{2}\right)}\right) \\
& \times e_{\alpha \ominus z_{1} \beta \ominus z_{2}}\left(t_{1}, t_{2}, a, b\right) \\
= & \frac{e_{\ominus z_{1} \ominus z_{2}}(a, b, 0,0)}{\left(\alpha-z_{1}\right)\left(\beta-z_{2}\right)}\left(\alpha \ominus z_{1}\right)\left(\beta \ominus z_{2}\right) e_{\alpha \ominus z_{1} \beta \ominus z_{2}}\left(t_{1}, t_{2}, a, b\right) .
\end{aligned}
$$

Then

$$
\begin{aligned}
& L_{t_{1}} L_{t_{2}}\left[H_{a, b}\left(t_{1}, t_{2}\right) f\left(t_{1}, t_{2}, a, b\right)\right]\left(z_{1}, z_{2}\right) \\
& =\iint_{0}^{\infty} e_{\ominus z_{1} \ominus z_{2}}\left(\sigma\left(t_{1}\right), \sigma\left(t_{2}\right), 0,0\right) H_{a, b}\left(t_{1}, t_{2}\right) e_{\alpha \oplus \beta} \\
& \quad \times\left(t_{1}, t_{2}, a, b\right) \Delta_{1} t_{1} \Delta_{2} t_{2} \\
& =\frac{e_{\ominus z_{1} \ominus z_{2}}(a, b, 0,0)}{\left(\alpha-z_{1}\right)\left(\beta-z_{2}\right)} \iint_{0}^{\infty} H_{a, b}\left(t_{1}, t_{2}\right) \\
& \times\left(\left(\alpha \ominus z_{1}\right)\left(\beta \ominus z_{2}\right)\right)\left(t_{1}, t_{2}\right) \\
& \times e_{\alpha \ominus z_{1} \beta \ominus z_{2}}\left(t_{1}, t_{2}, a, b\right) \\
& \times \Delta_{1} t_{1} \Delta_{2} t_{2}
\end{aligned}
$$




$$
\begin{gathered}
\begin{aligned}
\frac{e_{\ominus z_{1} \ominus z_{2}}(a, b, 0,0)}{\left(\alpha-z_{1}\right)\left(\beta-z_{2}\right)} \int_{b}^{\infty} \int_{a}^{\infty} & \left(\left(\alpha \ominus z_{1}\right)\left(\beta \ominus z_{2}\right)\right) \\
& \times e_{\alpha \ominus z_{1} \beta \ominus z_{2}}\left(t_{1}, t_{2}, a, b\right) \\
& \times \Delta_{1} t_{1} \Delta_{2} t_{2}
\end{aligned} \\
=\frac{e_{\ominus z_{1} \ominus z_{2}}(a, b, 0,0)}{\left(\alpha-z_{1}\right)\left(\beta-z_{2}\right)} \\
=e_{\ominus z_{1} \ominus z_{2}}(a, b, 0,0) L_{t_{1}} L_{t_{2}}\left[e_{\alpha \oplus \beta}\left(t_{1}, t_{2}, 0,0\right)\right] .
\end{gathered}
$$

Theorem 12. Let $f\left(t_{1}, t_{2}\right): \mathrm{T}_{1} \times \mathrm{T}_{2} \rightarrow \mathbb{C}$ and suppose that $f^{\Delta_{i}}$ and $f^{\Delta_{j}}$ are continuous for all $i=1,2, \ldots n$ and $j=$ $1,2, \ldots, m$, respectively; then the $\Delta$-double Laplace transforms for $f^{\Delta_{i}}$ and $f^{\Delta_{j}}$ are given by

$$
\begin{aligned}
& L_{t_{1}} L_{t_{2}}\left[f^{\Delta_{n}^{1}}\left(t_{1}, t_{2}\right)\right]\left(z_{1}, z_{2}\right) \\
& =z_{1}^{n} \overline{\bar{F}}\left(z_{1}, z_{2}\right)-z_{1}^{n-1} \bar{F}\left(0, z_{2}\right)-\cdots-\stackrel{-\Delta_{1}^{n-1}}{F}\left(0, z_{2}\right), \\
& L_{t_{1}} L_{t_{2}}\left[f^{\Delta_{m}^{2}}\left(t_{1}, t_{2}\right)\right]\left(z_{1}, z_{2}\right) \\
& \quad=z_{2}^{m} \overline{\bar{F}}\left(z_{1}, z_{2}\right)-z_{2}^{m-1} \bar{F}\left(z_{1}, 0\right)-\cdots-\stackrel{-\Delta_{2}^{m-1}}{F}\left(z_{1}, 0\right),
\end{aligned}
$$

for all $z_{1}, z_{2} \in \mathbb{C}$ with

$$
\begin{aligned}
& \lim _{t_{1} \rightarrow \infty}\left\{e_{\ominus z_{1} \ominus z_{2}}\left(t_{1}, t_{2}\right) f\left(t_{1}, t_{2}\right)\right\} \\
& =\lim _{t_{1} \rightarrow \infty}\left\{e_{\ominus z_{1} \ominus z_{2}}\left(t_{1}, t_{2}\right) f^{\Delta_{n}^{1}}\left(t_{1}, t_{2}\right)\right\} \cdots \\
& =\lim _{t_{1} \rightarrow \infty}\left\{e_{\ominus z_{1} \ominus z_{2}}\left(t_{1}, t_{2}\right) f^{\Delta_{n-1}^{1}}\left(t_{1}, t_{2}\right)\right\}=0, \\
& \lim _{t_{2} \rightarrow \infty}\left\{e_{\ominus z_{1} \ominus z_{2}}\left(t_{1}, t_{2}\right) f\left(t_{1}, t_{2}\right)\right\} \\
& \quad=\lim _{t_{2} \rightarrow \infty}\left\{e_{\ominus z_{1} \ominus z_{2}}\left(t_{1}, t_{2}\right) f^{\Delta_{m}^{2}}\left(t_{1}, t_{2}\right)\right\} \cdots \\
& \quad=\lim _{t_{2} \rightarrow \infty}\left\{e_{\ominus z_{1} \ominus z_{2}}\left(t_{1}, t_{2}\right) f^{\Delta_{m-1}^{2}}\left(t_{1}, t_{2}\right)\right\}=0,
\end{aligned}
$$

respectively.

Proof. Let $n=1$. On using the definition of the $\Delta$-single Laplace transform and integrating parts of the function $f^{\Delta_{1}^{1}}\left(t_{1}, t_{2}\right)$, we have

$$
\begin{aligned}
L_{t_{1}} & {\left[f^{\Delta_{1}^{1}}\left(t_{1}, t_{2}\right)\right]\left(z_{1}\right) } \\
& =\int_{0}^{\infty} e_{\ominus z_{1}}^{\sigma_{1}} f^{\Delta_{1}^{1}}\left(t_{1}, t_{2}\right) \Delta t_{1} \\
& =\left[f\left(t_{1}, t_{2}\right)\right]_{0}^{\infty}+z_{1} \int_{0}^{\infty} e_{\ominus z_{1}}^{\sigma_{1}} f\left(t_{1}, t_{2}\right) \Delta t_{1} \\
& =-f\left(0, t_{2}\right)+z_{1} \bar{F}\left(z_{1}, t_{2}\right) .
\end{aligned}
$$

Then the single Laplace transform with respect to $t_{1}$ is given by

$$
L_{t_{1}}\left[f^{\Delta_{1}^{1}}\left(t_{1}, t_{2}\right)\right]\left(z_{1}, z_{2}\right)=z_{1} \bar{F}\left(z_{1}, t_{2}\right)-f\left(0, t_{2}\right) .
$$

Similarly, Laplace transform for (44) with respect to $t_{2}$ is given by

$$
L_{t_{2}} L_{t_{1}}\left[f^{\Delta_{1}^{1}}\left(t_{1}, t_{2}\right)\right]\left(z_{1}\right)=z_{1} \overline{\bar{F}}\left(z_{1}, z_{2}\right)-\bar{F}\left(0, z_{2}\right) .
$$

Then (45) is called the $\Delta$-double Laplace transform for the function $f^{\Delta_{1}^{1}}\left(t_{1}, t_{2}\right)$. Similarly, if $m=1$ in (41), the $\Delta$-double Laplace transform for the function $f^{\Delta_{1}^{2}}\left(t_{1}, t_{2}\right)$ is given by

$$
L_{t_{2}} L_{t_{1}}\left[f^{\Delta_{1}^{2}}\left(t_{1}, t_{2}\right)\right]\left(z_{1}, z_{2}\right)=z_{2} \overline{\bar{F}}\left(z_{1}, z_{2}\right)-\bar{F}\left(z_{1}, 0\right) \text {. }
$$

Now, in order to obtain the $\Delta$-double Laplace transform for the function

$$
f^{\Delta_{2}^{1}}\left(t_{1}, t_{2}\right)=\frac{\partial^{2} f\left(t_{1}, t_{2}\right)}{\Delta_{1} t_{1}^{2}}
$$

with respect to $t_{1}, t_{2}$, we proceed as follows: first of all by taking the $\Delta$-single Laplace transform with respect to the variable $t_{1}$ and on using the fact that

$$
\begin{gathered}
\lim _{t_{1} \rightarrow \infty}\left\{e_{\ominus z_{1}}\left(t_{1}\right) f^{\Delta_{1}^{1}}\left(t_{1}, t_{2}\right)\right\}=0, \\
\lim _{t_{1} \rightarrow \infty}\left\{e_{\ominus z_{1}}\left(t_{1}\right) f\left(t_{1}, t_{2}\right)\right\}=0,
\end{gathered}
$$

we have

$$
\begin{aligned}
L_{t_{1}} & {\left[f^{\Delta_{2}^{1}}\left(t_{1}, t_{2}\right)\right]\left(z_{1}\right) } \\
& =\left[f^{\Delta_{1}^{1}}\left(t_{1}, t_{2}\right)\right]_{0}^{\infty}+z_{1} \int_{0}^{\infty} e_{\ominus z_{1}}^{\sigma_{1}} f^{\Delta_{1}^{1}}\left(t_{1}, t_{2}\right) \Delta t_{1} \\
& =-f^{\Delta_{1}^{1}}\left(0, t_{2}\right)+z_{1} \int_{0}^{\infty} e_{\ominus z_{1}}^{\sigma_{1}} f^{\Delta_{1}^{1}}\left(t_{1}, t_{2}\right) \Delta t_{1} .
\end{aligned}
$$

Thus on using (44), we obtain that

$$
\begin{aligned}
L_{t_{1}} & {\left[f^{\Delta_{2}^{1}}\left(t_{1}, t_{2}\right)\right]\left(z_{1}\right) } \\
& =z_{1}^{2} \bar{F}\left(z_{1}, t_{2}\right)-z_{1} \bar{F}\left(0, t_{2}\right)-f^{\Delta^{1}}\left(0, t_{2}\right) .
\end{aligned}
$$

In a similar way, the single Laplace transform with respect to $t_{2}$ is given by

$$
\begin{aligned}
& L_{t_{2}} L_{t_{1}}\left[f^{\Delta_{2}^{1}}\left(t_{1}, t_{2}\right)\right]\left(z_{1}, z_{2}\right) \\
& \quad=z_{1}^{2} \overline{\bar{F}}\left(z_{1}, z_{2}\right)-z_{1} \bar{F}\left(0, z_{2}\right)-\bar{F}^{\Delta^{1}}\left(0, z_{2}\right) .
\end{aligned}
$$

Further, the $\Delta$-double Laplace transform for the function $f^{\Delta_{2}^{2}}\left(t_{1}, t_{2}\right)=\partial^{2} f\left(t_{1}, t_{2}\right) / \Delta_{2} t_{2}^{2}$ with respect to $t_{1}, t_{2}$ is given by

$$
\begin{aligned}
& L_{t_{2}} L_{t_{1}}\left[f^{\Delta_{2}^{2}}\left(t_{1}, t_{2}\right)\right]\left(z_{1}, z_{2}\right) \\
& \quad=z_{2}^{2} \overline{\bar{F}}\left(z_{1}, z_{2}\right)-z_{2} \bar{F}\left(z_{1}, 0\right)-\bar{F}^{\Delta^{1}}\left(z_{1}, 0\right) .
\end{aligned}
$$

Finally, note that we can generalize the proof for $n, m$. 
Next, we define the double convolution of two time scale functions as follows.

Definition 13. The double convolution of the function $f, g$ : $\mathbf{T}_{1} \times \mathbf{T}_{2} \rightarrow \mathbb{R}$ is given by

$$
(f * * g)\left(t_{1}, t_{2}\right)=\iint_{0}^{t_{1}} f(\zeta, \eta) g\left(t_{1}, t_{2}, \sigma_{1}(\zeta), \sigma_{2}(\eta)\right) \Delta \zeta \Delta \eta
$$

In the next theorem we prove the properties of the double convolution on a time scale as follows.

Theorem 14. Let $f, g, h: \mathbf{T}_{1} \times \mathbf{T}_{2} \rightarrow \mathbb{R}$ be integrable functions on a time scale. Then the following properties are satisfied:

$$
\begin{aligned}
(f * * g)\left(t_{1}, t_{2}\right) & =(g * * f)\left(t_{1}, t_{2}\right), \\
(f * * g) * * h & =f * *(g * * h) .
\end{aligned}
$$

Proof. We can easily show that convolution is commutative by using the definition of the double convolution

$$
\begin{aligned}
(f & * * g)\left(t_{1}, t_{2}\right) \\
& =\iint_{0}^{t_{1}} f(\zeta, \eta) g\left(t_{1}, t_{2}, \sigma_{1}(\zeta), \sigma_{2}(\eta)\right) \Delta \zeta \Delta \eta \\
& =\iint_{0}^{t_{1}} f\left(t_{1}, t_{2}, \sigma_{1}(\zeta), \sigma_{2}(\eta)\right) g(\zeta, \eta) \Delta \zeta \Delta \eta .
\end{aligned}
$$

For the proof of the associative law, we also use the definition as follows:

$$
\begin{aligned}
& (f * *) * * h \\
& =\iint_{0}^{t_{1}}(f * * g)\left(t_{1}, t_{2}, \sigma_{1}(\zeta), \sigma_{2}(\eta)\right) h(\zeta, \eta) \Delta \zeta \Delta \eta \\
& =\iint_{0}^{t_{1}} \int_{\sigma_{2}(\eta)}^{t_{1}} \int_{\sigma_{1}(\zeta)}^{t_{1}} f\left(t_{1}, t_{2}, \sigma_{1}(\alpha), \sigma_{2}(\beta)\right) \\
& \quad \times g\left(\alpha, \beta, \sigma_{1}(\zeta), \sigma_{2}(\eta)\right) \\
& \quad \times h(\zeta, \eta) \Delta \zeta \Delta \eta \Delta \alpha \Delta \beta \\
& =\iint_{0}^{t_{1}} f\left(t_{1}, t_{2}, \sigma_{1}(\alpha), \sigma_{2}(\beta)\right)(f * * g)(\alpha, \beta) \Delta \alpha \Delta \beta \\
& \quad \times f * *(g * * h)\left(t_{1}, t_{2}\right) .
\end{aligned}
$$

Theorem 15 (convolution theorem). Let $f, g: \mathbf{T}_{1} \times \mathbf{T}_{2} \rightarrow \mathbb{C}$ be regulated functions. Then the double Laplace transform of the double convolution is given by

$$
L_{t_{2}} L_{t_{1}}\left[(f * * g)\left(t_{1}, t_{2}\right) ; z_{1}, z_{2}\right]=F\left(z_{1}, z_{2}\right) G\left(z_{1}, z_{2}\right) \text {. }
$$

Proof. By using the definition of the double Laplace transform on a time scale, we obtain that

$$
\begin{aligned}
& L_{t_{2}} L_{t_{1}}\left[(f * * g)\left(t_{1}, t_{2}\right) ; z_{1}, z_{2}\right] \\
& =\iint_{0}^{\infty} e_{\ominus z_{1} \ominus z_{2}}^{\sigma_{1} \sigma_{2}}\left(t_{1}, t_{2}, 0,0\right)\left[(f * * g)\left(t_{1}, t_{2}\right)\right] \Delta_{1} t_{1} \Delta_{2} t_{2} \\
& =\iint_{0}^{\infty}\left[\iint_{0}^{t_{1}} f(\zeta, \eta) g\left(t_{1}, t_{2}, \sigma_{1}(\zeta), \sigma_{2}(\eta)\right)\right. \\
& \left.\quad \times \Delta \zeta \Delta \eta e_{\ominus z_{1} \ominus z_{2}}^{\sigma_{1} \sigma_{2}}\left(t_{1}, t_{2}, 0,0\right)\right] \Delta_{1} t_{1} \Delta_{2} t_{2} \\
& =\iint_{0}^{\infty} f(\zeta, \eta)\left[\int_{\sigma_{1}(\zeta)}^{\infty} \int_{\sigma_{2}(\eta)}^{\infty} g\left(t_{1}, t_{2}, \sigma_{1}(\zeta), \sigma_{2}(\eta)\right)_{\ominus z_{1} \ominus z_{2}}^{\sigma_{1} \sigma_{2}}\right. \\
& \left.\times\left(t_{1}, t_{2}, 0,0\right) \Delta_{1} t_{1} \Delta_{2} t_{2}\right] \Delta \zeta \Delta \eta \\
& =\iint_{0}^{\infty} f(\zeta, \eta)\left[L _ { t _ { 2 } } L _ { t _ { 1 } } \left[H_{\sigma_{1}(\zeta), \sigma_{2}(\eta)}\left(t_{1}, t_{2}\right)\right.\right. \\
& \left.\left.\times g\left(t_{1}, t_{2}, \sigma_{1}(\zeta), \sigma_{2}(\eta)\right)\right]\right] \Delta \zeta \Delta \eta
\end{aligned}
$$

where $H_{\sigma_{1}(\zeta), \sigma_{2}(\eta)}\left(t_{1}, t_{2}\right)=H_{\sigma_{1}(\zeta),}\left(t_{1}\right) \otimes H_{\sigma_{2}(\zeta),}\left(t_{2}\right)$ and the symbol $\otimes$ denotes the tensor product. Thus we have

$$
\begin{aligned}
& L_{t_{2}} L_{t_{1}}\left[(f * * g)\left(t_{1}, t_{2}\right) ; z_{1}, z_{2}\right] \\
& \quad=\iint_{0}^{\infty} f(\zeta, \eta)\left[G\left(z_{1}, z_{2}\right)_{\ominus z_{1} \ominus z_{2}}^{\sigma_{1} \sigma_{2}}(\zeta, \eta, 0,0)\right] \Delta \zeta \Delta \eta \\
& \quad=G\left(z_{1}, z_{2}\right) \iint_{0}^{\infty} f(\zeta, \eta)_{\ominus z_{1} \ominus z_{2}}^{\sigma_{1} \sigma_{2}}(\zeta, \eta, 0,0) \Delta \zeta \Delta \eta \\
& \quad=F\left(z_{1}, z_{2}\right) G\left(z_{1}, z_{2}\right) .
\end{aligned}
$$

Finally, we give the solution to the wave equation in one dimension as follows. Consider the wave equation in the form of

$$
u^{\Delta_{1} \Delta_{1}}-u^{\Delta_{2} \Delta_{2}}=0
$$

under the conditions

$$
\begin{gathered}
u(x, 0)=e_{1}(x), u(0, t)=e_{1}(t), \\
u^{\Delta_{2}}(0, t)=e_{1}(t), u^{\Delta_{1}}(x, 0)=e_{1}(x) .
\end{gathered}
$$

Then by formally taking the double Laplace transform, we get

$$
\begin{aligned}
L_{t_{2}} L_{t_{1}}\left[u(t, x) ; z_{1}, z_{2}\right] & \\
& =\frac{z_{1}+1}{\left(z_{2}-1\right)\left(z_{1}^{2}-z_{2}^{2}\right)}-\frac{z_{2}+1}{\left(z_{1}-1\right)\left(z_{1}^{2}-z_{2}^{2}\right)} .
\end{aligned}
$$


By using partial fractions, we have

$$
\begin{aligned}
L_{t_{2}} L_{t_{1}}\left[u(t, x) ; z_{1}, z_{2}\right] \\
=\frac{1}{2\left(z_{2}-1\right)\left(z_{1}-z_{2}\right)}+\frac{1}{2\left(z_{2}-1\right)\left(z_{1}+z_{2}\right)} \\
\quad+\frac{1}{2 z_{2}\left(z_{2}-1\right)\left(z_{1}-z_{2}\right)}-\frac{1}{2 z_{2}\left(z_{2}-1\right)\left(z_{1}+z_{2}\right)} \\
\quad-\frac{1}{2\left(z_{1}-1\right)\left(z_{1}-z_{2}\right)}+\frac{1}{2\left(z_{1}-1\right)\left(z_{1}+z_{2}\right)} \\
\quad-\frac{1}{2 z_{1}\left(z_{1}-1\right)\left(z_{1}-z_{2}\right)}-\frac{1}{2 z_{1}\left(z_{1}-1\right)\left(z_{1}+z_{2}\right)} .
\end{aligned}
$$

Thus the solution to the above equation is given by

$$
u(t, x)=e_{1 \oplus 1}(t, x, 0,0) .
$$

In particular, if we consider the case $\mathbf{T}_{1} \times \mathbf{T}_{2}=\mathbb{R} \times \mathbb{R}, \sigma(t)=$ $t, \sigma(x)=x$, for all $(t, x) \in \mathbf{T}_{1} \times \mathbf{T}_{2}$, then $e_{a \oplus b}(t, x, 0,0)=$ $e^{a x+b t}$ for any constant $a, b \in \mathbb{R}$ and so the solution is given by

$$
u(t, x)=e^{t+x}
$$

\section{Conflict of Interests}

The authors declare that they have no conflict of interests regarding the publication of paper.

\section{Acknowledgment}

The authors are grateful to the referees for the useful comments regarding presentation in the early version of the paper. The authors also gratefully acknowledge that this project was supported by King Saud University, Deanship of Scientific Research College of Science Research Center.

\section{References}

[1] H. A. Agwa, F. M. Ali, and A. Kilıçman, "A new integral transform on time scales and its applications," Advances in Difference Equations, vol. 2012, article 60, 2012.

[2] M. Bohner and A. Peterson, "Laplace transform and Ztransform: unification and extension," Methods and Applications of Analysis, vol. 9, no. 1, pp. 155-162, 2002.

[3] J. M. Davis, I. A. Gravagne, B. J. Jackson, R. J. Marks, and A. A. Ramos, "The Laplace transform on time scales revisited," Journal of Mathematical Analysis and Applications, vol. 332, no. 2, pp. 1291-1306, 2007.

[4] R. P. Agarwal, C. Ahlbrandt, M. Bohner, and A. Peterson, "Discrete linear Hamiltonian systems: a survey," Dynamic Systems and Applications, vol. 8, pp. 307-333, 1999.

[5] R. P. Agarwal and M. Bohner, "Quadratic functionals for second order matrix equations on time scales," Nonlinear Analysis: Theory, Methods and Applications, vol. 33, no. 7, pp. 675-692, 1998.
[6] R. P. Agarwal and M. Bohner, "Basic calculus on time scales and some of its applications," Results in Mathematics, vol. 35, pp. 322, 1999.

[7] C. D. Ahlbrandt, M. Bohner, and J. Ridenhour, "Hamiltonian systems on time scales," Journal of Mathematical Analysis and Applications, vol. 250, no. 2, pp. 561-578, 2000.

[8] L. Erbe and S. Hilger, "Sturmian theory on measure chains," Differential Equations and Dynamical Systems, vol. 1, pp. 223246, 1993.

[9] M. Bohner and G. S. Guseinov, "The convolution on time scales," Abstract and Applied Analysis, vol. 2007, Article ID 58373, 24 pages, 2007.

[10] B. Jackson, "Partial dynamic equations on time scales," Journal of Computational and Applied Mathematics, vol. 186, no. 2, pp. 391-415, 2006. 


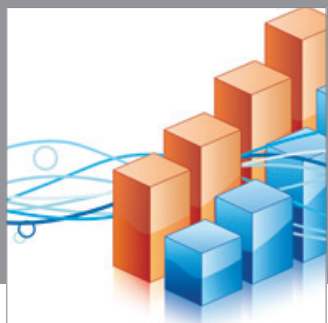

Advances in

Operations Research

mansans

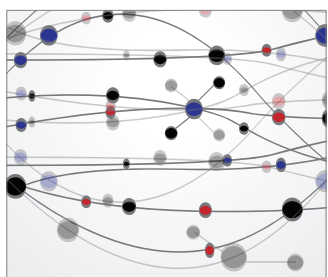

The Scientific World Journal
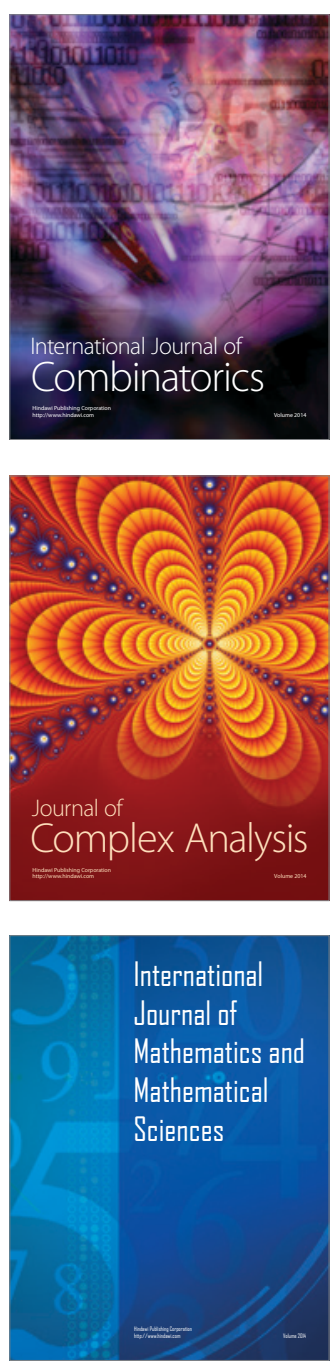
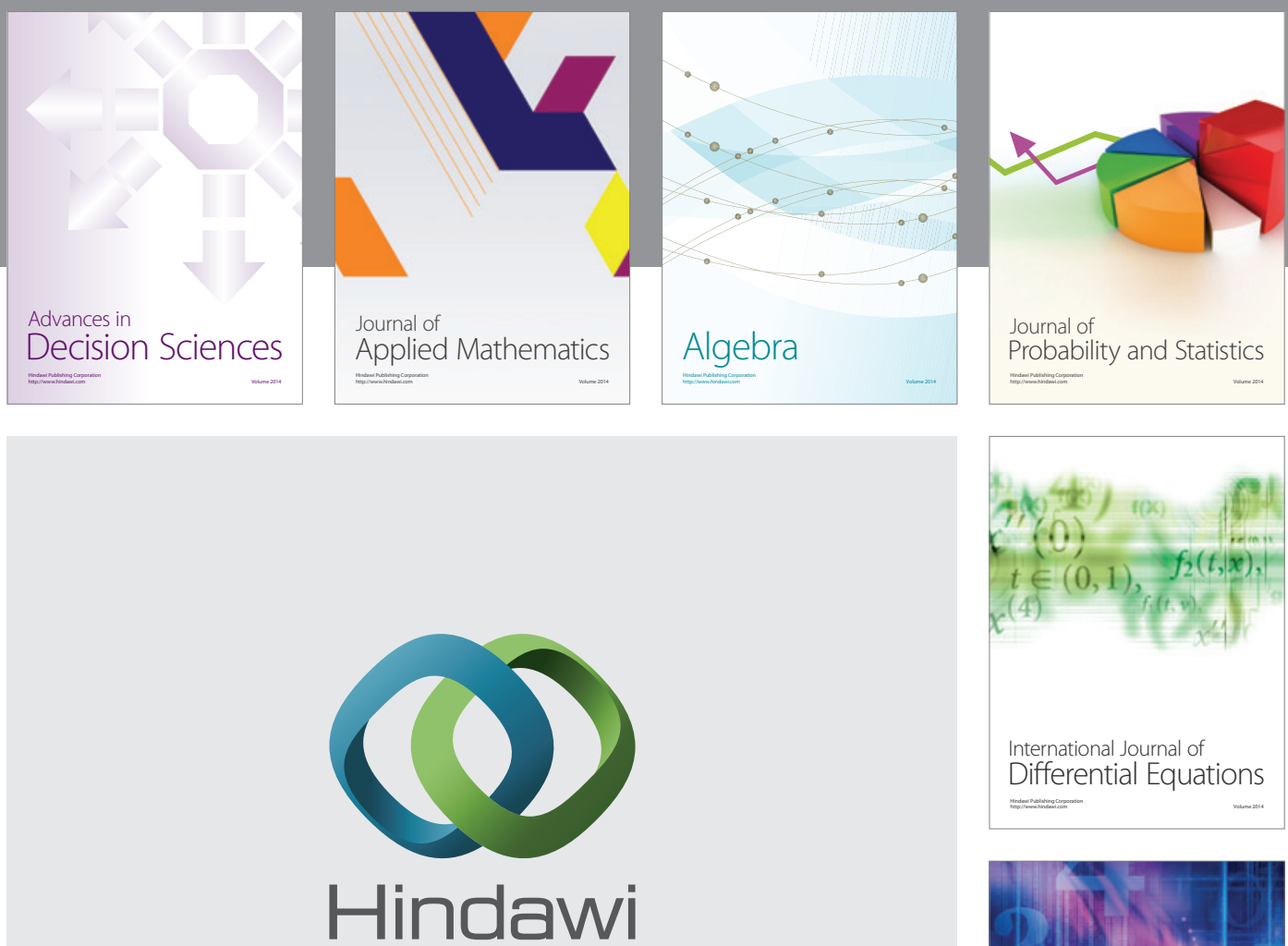

Submit your manuscripts at http://www.hindawi.com
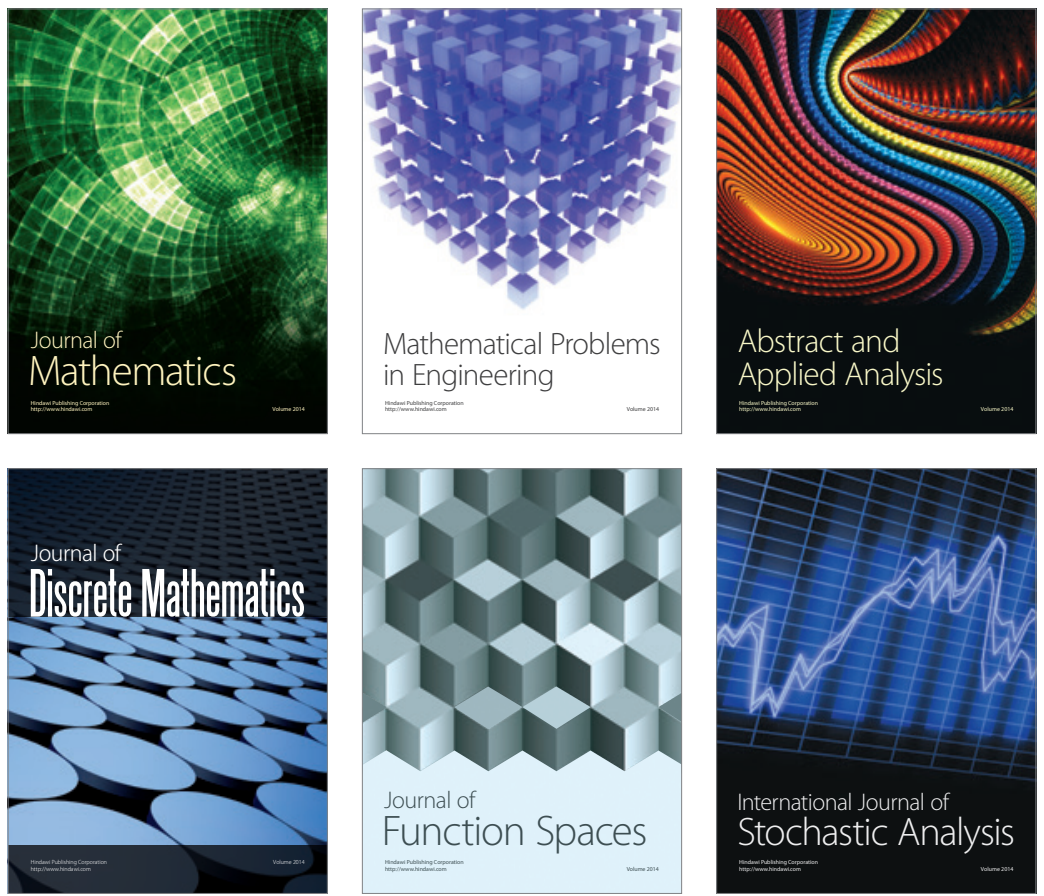

Journal of

Function Spaces

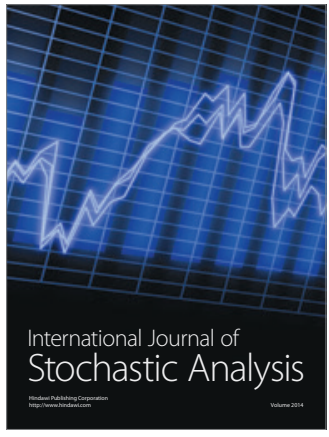

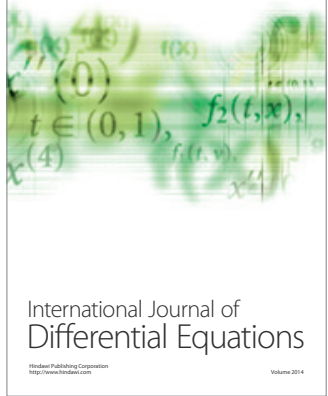
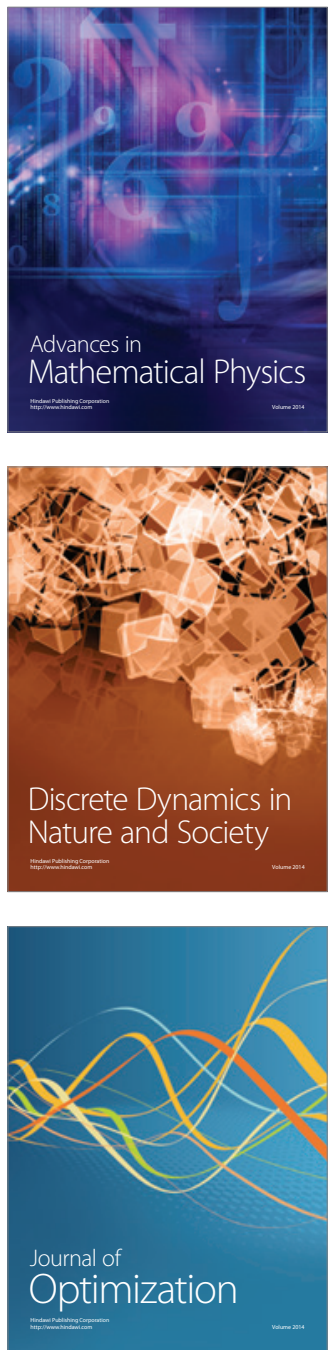